\title{
Coronary vasospasm-induced periodic ventricular fibrillation and successful ablation through coronary stenting
}

\author{
Kamil Gülşen ${ }^{1}$, Burak Ayça², Levent Cerit ${ }^{1}$, Ertuğrul Okuyan ${ }^{2}$ \\ ${ }^{1}$ Department of Cardiology, Near East University School of Medicine, Nicossia, Turkey \\ ${ }^{2}$ Department of Cardiology, Bagcilar Education and Research Hospital, Istanbul, Turkey
}

Postep Kardiol Inter 2015; 11, 4 (42): 337-340 DOI: $10.5114 /$ pwki.2015.55608

\section{Introduction}

Coronary vasospasm-induced ventricular fibrillation (VF) is a well-documented clinical condition, and there are existing reports in medical publications on this subject [1]. Treatment approaches for these patients can be challenging. In most cases, vasodilator therapy relieves anginal and VF episodes, but sometimes, in the case of recurrence, intracardiac defibrillator (ICD) implantation is performed with concomitant medical therapy [2]. When a patient receives multiple ICD shocks for recurrent VF episodes after vasospastic attacks in spite of the use of vasodilator therapy, it is an undesirable situation for both the patient and the doctor.

In this paper, we present a case of a patient suffering from this clinical condition that was successfully treated through coronary stent placement.

\section{Case report}

A 59-year old male patient was referred to our hospital after suffering a VF attack and was successfully treated with defibrillation. At his initial evaluation, the patient's mind was clear and his hemodynamic parameters and other physical examination findings were normal. His electrocardiogram results indicated a sinus rhythm with a rate of $60 \mathrm{bpm}$, there was no significant ischemic finding, the QT interval was normal and there was no suggestion of Brugada syndrome or arrhythmogenic right ventricular dysplasia (Figure $1 \mathrm{~A}$ ). Biochemical tests including cardiac troponin, serum potassium and magnesium levels were all normal. His previous medical history indicated that there had been two syncopal episodes within the last 2 months. There were no specific triggers for these episodes, but the patient described anginal chest pain immediately before the syncopal attacks. He had experienced no exercise-induced angina, and there were no instances in his family history of sudden death of unknown origin. His echocardiogram results showed that the cardiac chambers were of a normal size, there was normal systolic function of both left and right ventricles, and the wall thickness was also normal. Coronary angiography was administered and a moderate lesion was detected in the middle portion of the right coronary artery (RCA) (Figures $2 \mathrm{~A}-\mathrm{C}$ ). This lesion was evaluated with fractional flow reserve (FFR) using $200 \mu \mathrm{g}$ and then $300 \mu \mathrm{g}$ intravenous adenosine injections. The FFR ratio was found to be 0.86 . Amiodarone perfusion was administered at the time of his first medical contact, and this treatment was continued for $24 \mathrm{~h}$. The day after hospitalization he experienced another VF episode and was successfully treated with defibrillation. His monitor records detected an ST elevation in inferior leads a few seconds before the VF (Figure $1 \mathrm{~B}$ ). Vasodilator treatment including nitrate and calcium channel blockers (nifedipine, isosorbide mononitrate) was added to his therapy. Despite vasodilator therapy being applied, he experienced a similar episode 1 day after (Figure $1 \mathrm{C}$ ), and therefore an ICD was implanted in the patient. Intravenous nitrate infusion commenced and was titrated to the maximum dosage that the patient's blood pressure would allow. In addition, the nifedipine dosage was increased to $60 \mathrm{mg} /$ day. A second calcium channel blocker agent was not added. Over the following 2 days, the ICD device shocked the patient due to similar episodes (Figures $1 \mathrm{D}, \mathrm{E}$ ). As the vasodilatator therapy alone failed to prevent the VF attacks, the decision was made to stent the suspected culprit lesion. A provocation test was not performed due to the risk of severe vasospasm and a fatal arrhythmic storm. A long-

\section{Corresponding author:}

Kamil Gülşen, Department of Cardiology, Near East University School of Medicine, Heart Centre, 99138 Nicossia, Turkey, phone: +900548 872 9539, e-mail: kamilgulsen2000@yahoo.com

Received: 10.11 .2014 , accepted: 8.04.2015. 

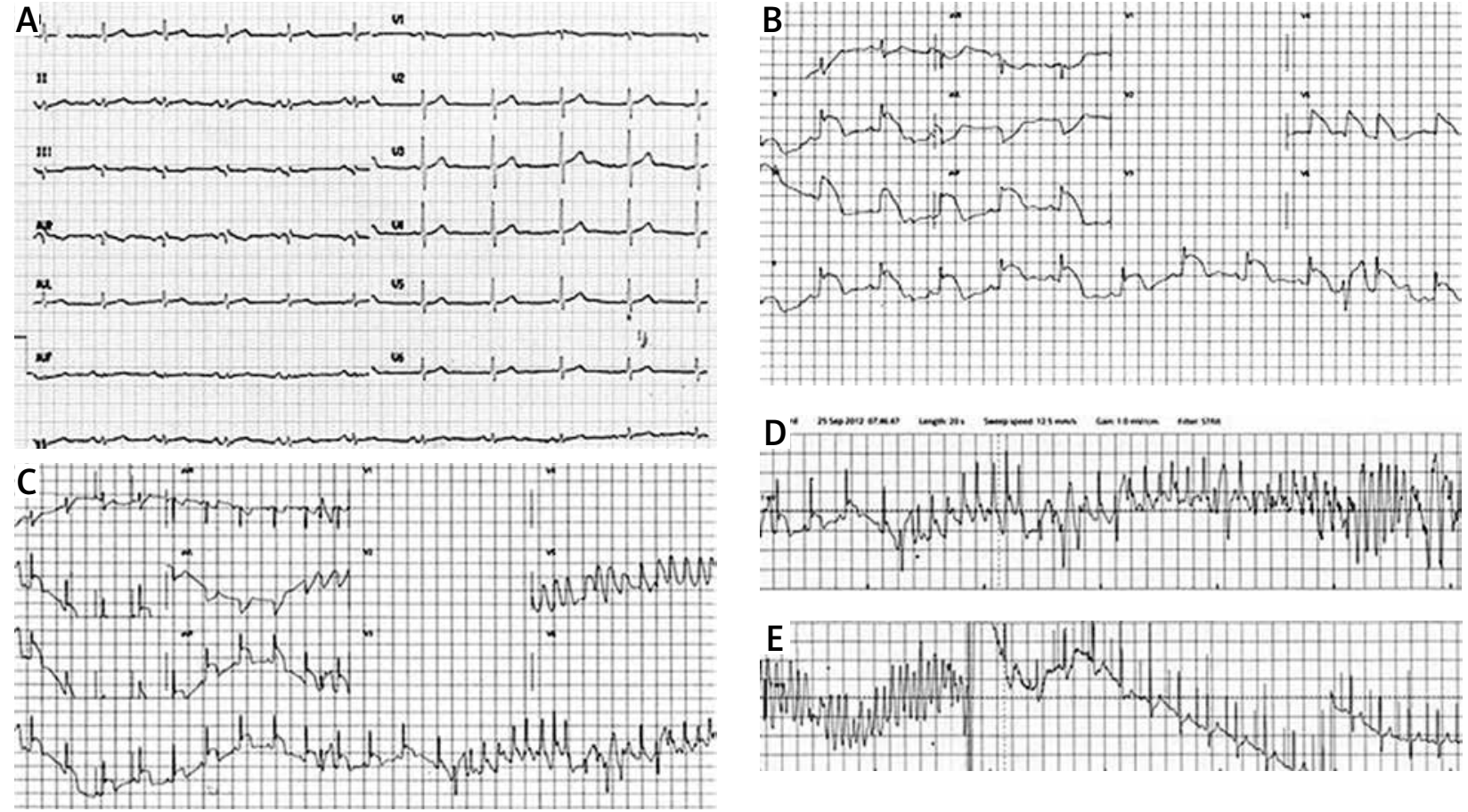

Figure 1. A - Initial electrocardiogram. B - ST elevation in inferior leads during vasospasm. C - VF following ST elevation. D - defibrillation and maintaining sinus rhythm

length new-generation drug-eluting stent (DES) (Xience $\mathrm{V}$ $3.5 \times 28 \mathrm{~mm}$ ) was preferred to completely cover the vasospastic segment in the RCA (Figure $2 \mathrm{D}$ ). Vasodilatator therapy was continued with both $60 \mathrm{mg}$ of isosorbide- 5 mononitrate and $60 \mathrm{mg}$ of long-acting nifedipine administered once a day. After the coronary stenting, VF attacks did not reoccur, and the patient has now been free from any anginal and VF attacks for 2 years.

\section{Discussion}

Vasospastic angina-induced malignant ventricular arrhythmias play a significant role in sudden cardiac death etiology [3], but there is no consensus on the management strategy for these patients. Even though, according to some reports, vasodilatator therapy alone is sufficient in such cases, there are other opinions that in some cases ICD implantation is a necessity [4, 5]. In addition, Gaspardone et al. stated that stent placement for the coronary vasospasm could lead to satisfactory results in selected patients [6]. Stenting could be considered in cases where vasospasm-related symptoms could not be kept under control using optimal medication. On the other hand, according to a study by Nishizaki et al., patients in whom coronary vasospasm emerged with ventricular arrhythmia have vulnerable myocardial substrate and always have a risk of sudden cardiac death [7]. Therefore, an ICD could be considered as a treatment option even after the patient experiences their first attack. In our case, neither vasodilator therapy nor the ICD solved the problem.
Coronary vasospasm could occur several times in different vessels and segments and could cause diffuse narrowing [8]. Tsujita et al. demonstrated intimal hyperplasia and thick fibrous atheroma plaque in the vasospastic segment with intravascular ultrasound (IVUS) [9]. Also, two thirds of patients have noncritical atherosclerotic plaque in the vasospastic segment [10]. Therefore if there is a noncritical plaque in the region of the vasospastic vessel, this could help clinicians to attempt correct localization. There are various factors underlying the pathophysiology of the coronary vasospasm. These precipitate local hyperreactivity of the coronary smooth muscle to a variety of constrictor stimuli that act through various surface receptors [10]. Endothelial dysfunction in non-critic plaque could facilitate the vasospasm. Despite optimal medical treatment with calcium channel blockers and nitrates, $5 \%$ to $30 \%$ of patients continue to have recurrent anginal episodes, and both myocardial infarction and arrhythmic sudden cardiac death may occur [11].

Although there are similar case reports in the medical literature [12], the case that we have presented differs from others due to its periodic nature. Data are scarce in current publications with respect to the optimal management method for cases where periodic vasospasm and VF attacks occur despite the use of appropriate vasodilatator therapy. There are some studies demonstrating altered endothelial functions after placement of the first generation DES [13]. Kounis et al. stated that some DES thromboses could be related to hypersensitivity and coronary vasospasm caused by coronary stents [14]. How- 

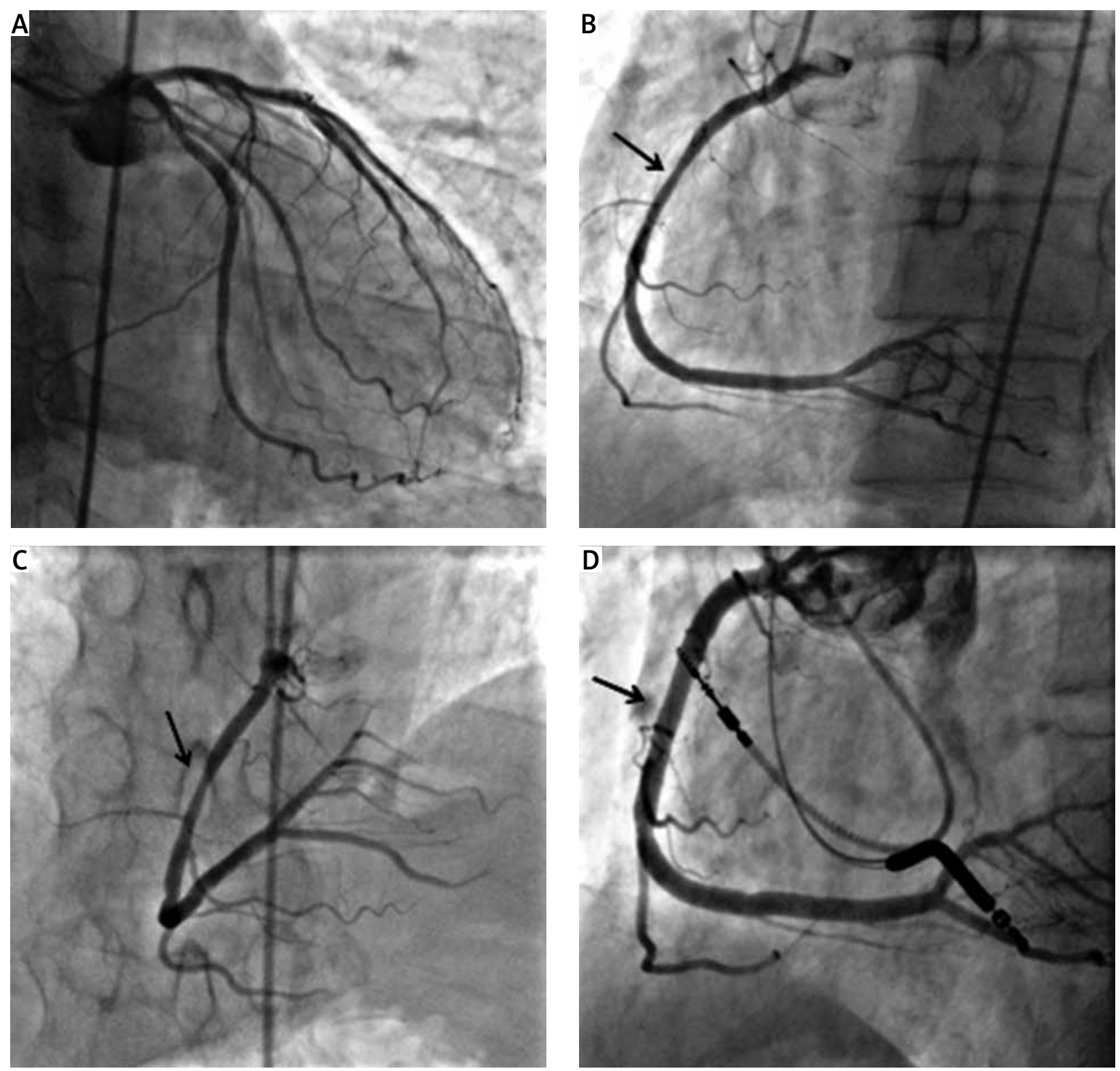

Figure 2. A, B, C-Coronary angiography of the patient. D-RCA after coronary stenting

ever, third generation DES polymers are more compatible with vascular endothelium. Also, as there has been no study demonstrating that coronary vasospasm is induced by a third generation DES, we elected to use one in this case. Although coronary stenting is not a widespread treatment modality for vasospasm, in our case the presence of noncritical plaque in the RCA suggested that stent insertion may solve the problem. As the patient has been free from any VF attacks for 2 years since coronary stenting, this has validated our opinion. Also, this is the first reported case of coronary vasospasm treated with a third generation DES. As we have noted above, a DES can treat vasospasm as well as cause vasospasm; however, we believe that our case could help clinicians in their decision making under such challenging conditions.

\section{Conclusions}

Coronary vasospasm-induced malignant ventricular arrhythmia should always be considered in cases of sudden cardiac death where the ventricular arrhythmia's etiology is not clear. Hence, recommended treatment could be percutaneous coronary intervention instead of electrophysiological study and radiofrequency ablation.

\section{Conflict of interest}

The authors declare no conflict of interest.

\section{References}

1. Myerburg RJ, Kessler KM, Mallon SM, et al. Life threatening ventricular arrhythmias in patients with silent myocardial ischemia 
due to coronary artery vasospasm. N Eng J Med 1992; 326 1451-5.

2. Meisel SR, Mazur A, Chetboun I, et al. Usefulness of implantable cardioverter-defibrillators in refractory variant angina pectoris complicated by ventricular fibrillation in patients with angiographically normal coronary arteries. Am J Cardiol 2002; 89: 1114-6.

3. Chevalier P, Dacosta A, Defaye P, et al. Arrhythmic cardiac arrest due to isolated coronary artery spasm: long-term outcome of seven resuscitated patients. J Am Coll Cardiol 1998; 31: 57-61.

4. Yamashina Y, Yagi T, Namekawa A, et al. Favorable outcomes of patients with vasospastic angina associated with cardiac arrest. J Cardiol 2014; 63: 41-5.

5. Matsue Y, Suzuki M, Nishizaki M, et al. Clinical implications of an implantable cardioverter-defibrillator in patients with vasospastic angina and lethal ventricular arrhythmia. J Am Coll Cardiol 2012; 60: 908-13.

6. Gaspardone A, Tomai F, Versaci F, et al. Coronary artery stent placement in patients with variant angina refractory to medical treatment. Am J Cardiol 1999; 84: 96-8, A8.

7. Nishizaki M, Arita M, Sakurada $H$, et al. Polymorphic ventricular tachycardia in patients with vasospastic angina: clinical and electrocardiographic characteristics and long-term outcome. Jpn Circ J 2001; 65: 519-25.

8. Kapoor JR, Price ET, Nijmeh JR, et al. Multivessel coronary artery spasm and cardiac arrest following single vessel stenting. Int J Cardiol 2008; 129: e35-6.

9. Tsujita K, Sakamoto K, Kojima S, et al. Coronary plaque component in patients with vasospastic angina: a virtual histology intravascular ultrasound study. Int J Cardiol 2013; 168: 2411-5.

10. Kaski JC, Crea F, Meran D, et al. Local coronary supersensitivity to diverse vasoconstrictive stimuli in patients with variant angina. Circulation 1986; 74: 1255-65.

11. Nakamura M, Takeshita A, Nose Y, et al. Clinical characteristics associated with myocardial infarction, arrhythmias, and sudden death inpatients with vasospastic angina. Circulation 1987; 75 : 1110-6.

12. Naqvi SY, Hanley A, Crowley J. Ventricular fibrillation due to coronary vasospasm. BMJ Case Rep 2014; 2014: pii: bcr2013203253.

13. Togni $M$, Windecker F, Cocchia R, et al. Sirolimus-eluting stents associated with paradoxic coronary vasoconstriction. J Am Coll Cardiol 2005; 46: 231-6.

14. Kounis GN, Hahalis G, Soufras GD, et al. Kounis syndrome and simultaneous multivessel acute coronary syndromes after successful drug-eluting stent implantation. Int J Cardiol 2008; 127 : 146-8. 